\title{
Modeling and Analysis of Drift Error in a MSSG with Double Spherical Envelope Surfaces
}

\author{
Xin Chaojun ${ }^{1}$, Cai Yuanwen ${ }^{1}$, Ren Yuan ${ }^{1 *}$, and Fan Yahong ${ }^{2}$ \\ ${ }^{1}$ Equipment Academy, Beijing 101416, China \\ ${ }^{2}$ Beijing Institute of Control Engineering, Beijing 100262, China
}

(Received 16 December 2015, Received in final form 30 May 2016, Accepted 14 June 2016)

\begin{abstract}
To improve the sensing accuracy of the newly developed magnetically suspended sensitive gyroscope (MSSG), it is necessary to analyze the causes of drift error. This paper build the models of disturbing torques generated by stator assembly errors based on the geometric construction of the MSSG with double spherical envelope surfaces, and further reveals the generation mechanism of the drift error. Then the drift error from a single stator magnetic pole is calculated quantitatively with the established model, and the key factors producing the drift error are further discussed. It is proposed that the main approaches in reducing the drift error are guaranteeing the rotor envelope surface to be an ideal spherical and improving the controlling precision of rotor displacement. The common problems associated in a gyroscope with a spherical rotor can be effectively resolved by the proposed method.
\end{abstract}

Keywords : Magnetically suspended sensitive gyroscope (MSSG), drift error, disturbing torque, spherical rotor, modeling

\section{Introduction}

Active magnetic bearings (AMBs) have been successfully employed for many space missions as ideal actuator for the attitude control of the spacecraft [1-3], since they possess a variety of priority of zero friction, high-speed operation, active vibration suppression, long life span and great potential of high control accuracy. Traditionally, AMBs are employed as control moment gyroscopes, moment wheels or reaction wheels in space missions $[4$, 5]. In recent years, researchers have begun to develop inertial sensors based on AMBs, named magnetically suspended sensitive gyroscope (MSSG) [6]. MSSG is used to measure attitude angle or angular velocities. Compared to traditional mechanical gyroscopes, MSSG has a wide prospect of application in aerospace, aviation, navigation, homing, robots, and automobiles.

Y. Maruyama et al. raised the method of inertial measurement using AMBs [6]. They discussed the principles of two-axis angular velocity measurement by AMBs, and experimentally succeeded in using an industrial AMB

(C)The Korean Magnetics Society. All rights reserved.

*Corresponding author: Tel: +86-10-6636-4384

Fax: +86-10-6636-4198, e-mail: renyuan823@aliyun.com motor as a gyroscope. Their research proves the technical feasibility and shows the potential of high-precesion and high-bandwidth of MSSGs. However, due to lack of effective error compensation, the amplitude error is as high as $10 \%$ in the angular velocity measurement [6-8]. Fang J. et al. proposed a magnetically suspended control moment gyroscope, which can work in two modes of actuator and attitude sensor [9]. By a PID controller with cross-axis feedback compensation, the angular velocity couplings between the $x$ - and $y$-axes are decoupled. However, the errors of the rotor are not considered in these studies. The assembly errors and process errors of stator are also neglected. Such an estimation method leads to an inaccurate measurement result.

Unlike mechanical bearing gyroscopes, in a MSSG, the AMB itself is utilized as a rotating gyroscope, and the rotor is in the free state of levitation. Only the forces directly acting on the rotor may produce disturbing torques, and cause gyroscope drift errors. There are only three field forces of gravity, inertia force, magnetic force and resistance of the residual gas in the stator house that directly acting on the rotor in an AMB.

Especially in the MSSG studied in this paper, both the magnetic pole envelope surfaces (MPES) of stator and the rotor are spherical surfaces, so as to realize the dynamic 
decoupling in inertial measurement, thus the measurement precision can be improved. When the geometric centers of the ideal spherical envelope surfaces are coincident, and the device performance parameters of each channel are identical, the magnetic forces over the entire surface of the rotor will pass through its geometric center as it spinning steadily about its polar axis. On this occasion, there are no disturbing torques produced, and no drift errors generated. However, dues to the material inhomogeneity, machining, assembly precision, centrifugal deformation and some other reasons, the actual MPESs of stator and rotor will deviate from the ideal sphere [10]. The deviation makes magnetic forces away from the geometric center of the rotor, and disturbing torques acting on geometric center are produced, then the drift errors $[11,12]$. Therefore, it is necessary to build the models of disturbing torque for error compensation.

In recent years, disturbing torques have been deeply researched in superconducting gyroscopes. Chuan $\mathrm{He}$ preliminary analyzed the disturbing torques produced by the asphericity of rotor in a superconducting gyroscope [13]. Aspherical disturbing torques are lead into two categories. Nevertheless, the author only considers the disturbing torques produced by suspension coils in $Z$-axis direction, the other factors producing disturbing torque are not in-depth analyzed, nor the drift errors generated from the disturbing torque. Cui Chunyan analyzed disturbing torques produced by the asphericity of rotor, and deduced the model of drift rate in a superconducting gyroscope more systematically [14]. The author mainly focuses on the disturbing torques produced by the asphericity of rotor, but disturbing torques from errors of stator have not been sufficiently studied.

Based on the assumption of mass balance of the spherical rotor, we focus our attention on the mechanism and rules of the disturbing torque due to errors of stator in a MSSG. According to the physical mechanism, theoretical model of drift angular velocity from three kinds of assembly error are established. By substituting the MSSG parameters into the formula, the numerical results are provided and solutions of reducing drift error are pointed out.

\section{Disturbing Torques of the MSSG}

\subsection{Description of the MSSG system}

The MSSG studied in this paper consists of rotor, stator, high speed motor, torquer and a stator house. Figure 1 shows the configuration of the MSSG.

The rotor of the MSSG is spherical in structure. Radiuses of rotor MPESs in axial and radial are different, while the

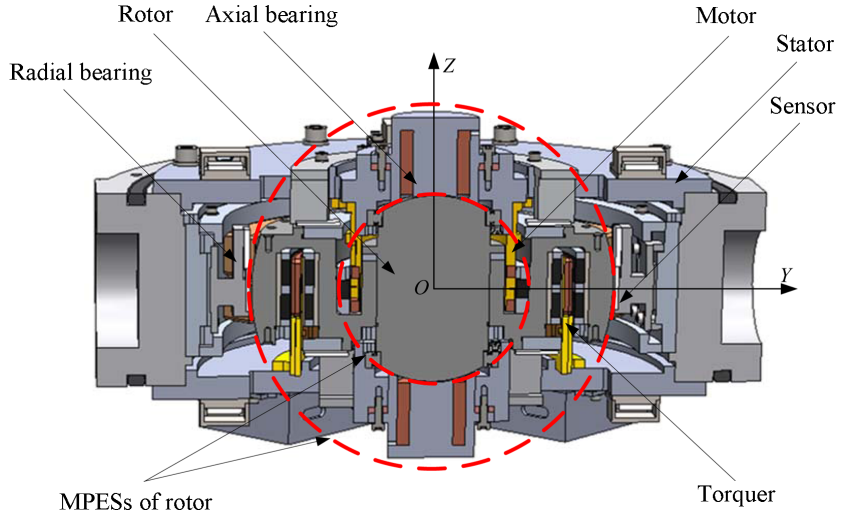

Fig. 1. (Color online) Configuration of the MSSG.

radius is $r_{1}$ in radial and $r_{2}$ in axial. And the rotor is made of magnetic material in whole.

There are three pairs of magnetic poles used as magnetic bearings on the stator of MSSG, named axial bearing, radial bearings in $X$-direction and $Y$-direction, respectively. Two pairs of radial bearing are symmetrically fixed along the coordinate axis in the $O X Y$-plane, as is shown in Fig. 2. The pair of axial bearing is symmetrically attached to the $Z+$ and $Z$-axis direction. Radius of MPES consisted by the two pairs of radial bearing is $R_{1}$, and that of MPES consisted by the pair of axial bearing is $R_{2}$. The two envelope surfaces of magnetic bearings constitute a spherical stator cavity, whose geometric center is coincident with centers of the two spherical MPESs in stator. The rotor is located in the center of the cavity when the system is running in an equilibrium state. The gap between the MPESs of the stator and rotor is $0.35 \mathrm{~mm}$ in nominal.

The rotation of the rotor is driven by a hollow cup non iron core permanent magnet brushless DC motor, whose coils are cured on the frame of the cup-chapped motor stator. The motor magnets are installed in the rotor of the

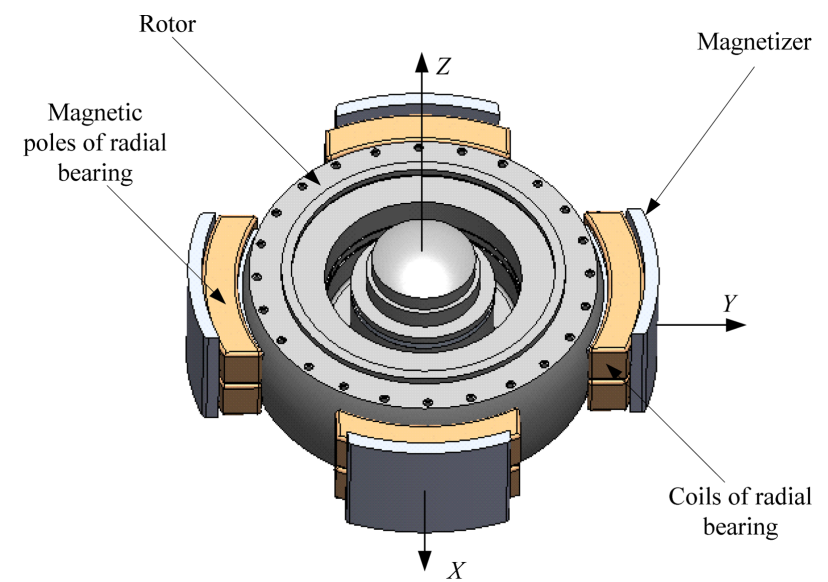

Fig. 2. (Color online) Configuration of the radial magnetic bearing. 


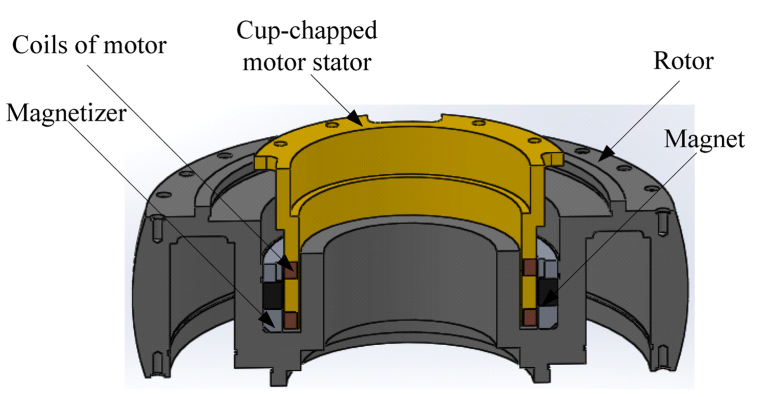

Fig. 3. (Color online) Cross-sectional structure of the motor.

MSSG with four pole structure. The built-in hall sensors are used to detect the rotate speed of MSSG rotor, and to realize reversing detection of the motor. The crosssectional structure of the motor is drawn in Fig. 3.

The levitation mode of the rotor can be described as follows. The axial and radial bearings are all electromagnets, therefore the control current would be provided through the coil on the poles, and corresponding magnetic field would be generated. The magnetic pull would act on the rotor made of soft magnetic material, pulling it diverge from its original position. However, by adjust the control currents in the coils of the electromagnet, the opposite magnetic pulls acting on the rotor in the same directions would be equilibrium, making the rotor levitating in the center of the stator cavity.

The torquer is a Lorentz-type magnetic bearing, which is used to control the tilting of the rotor. When the rotor is disturbed, its motion state will change. The eddy-current sensors are used to pick up the displacement signals of the rotor. Change in position of the rotor can be detected by the sensors. And the control system will apply corresponding control currents in different coils according to the sensors' signals, which will transform to magnetic

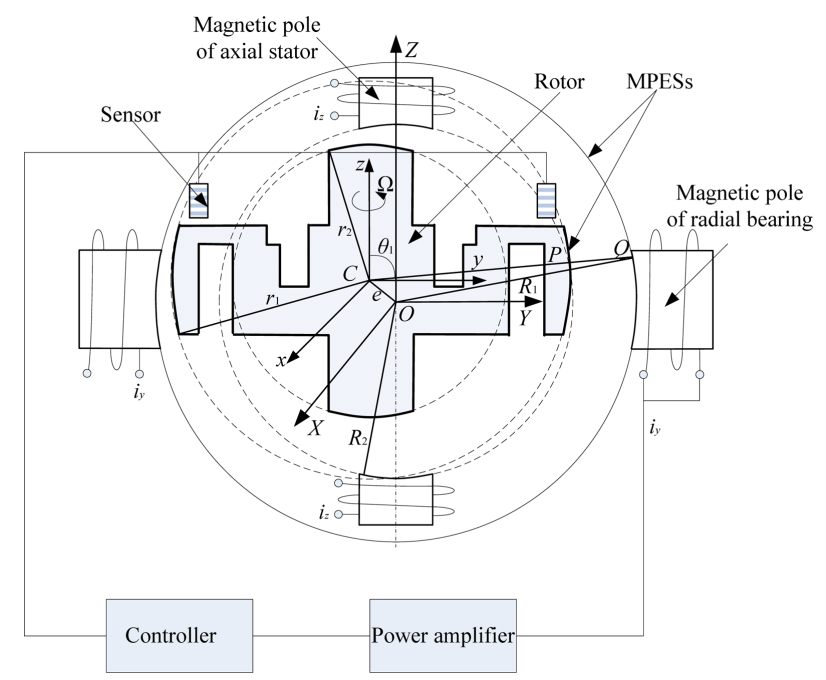

Fig. 4. (Color online) Block diagram for the MSSG system. pulls and impel the rotor back to its original position, as is shown in Fig. 4.

Aside from all the mechanical parts described above, the MSSG also consists of power amplifiers, signal conditioning circuits, and correlative filters.

\subsection{Modeling of gap between magnetic poles}

There are three coordinate systems were introduced in Fig. 4. In the rotor coordinate system $(x, y, z), C$ is defined as the geometric center of rotor, and also the origin of the rotor coordinate system. The axes are defined to be aligned with principal axes of inertia of rotor. And $\Omega$ is the spinning speed of the rotor around the major axis of inertia, $z$-axis. Origin of the stator coordinate $\operatorname{system}(X$, $Y, Z)$ is the center of the stator cavity. A spherical coordinate system $\left(R_{1}, \theta, \varphi\right)$ is introduced in correspond with the rectangular coordinate system $(X, Y, Z)$.

The unit vector of spinning axis of rotor $z_{0}$ can be written in the stator coordinate system as

$$
\boldsymbol{z}_{0}=\alpha \boldsymbol{X}_{0}+\beta \boldsymbol{Y}_{0}+\gamma \boldsymbol{Z}_{0}
$$

where $\alpha, \beta, \gamma$ are the direction cosines of $\boldsymbol{z}_{0}$ in the stator coordinate system, and $\boldsymbol{X}_{0}, \boldsymbol{Y}_{0}, \boldsymbol{Z}_{0}$ are the unit vectors of the axes in stator coordinate.

Assuming that point $P$ is on the spherical surface of the rotor, and the line $C P$ intersects the stator magnetic pole surface at point $Q$, the gap size between stator magnetic pole surface and the rotor can be written as

$$
\delta=|P Q|=|C Q|-|C P|
$$

In the case of minute offset, vector $\boldsymbol{C Q}, \boldsymbol{O Q}$ are approximately the same direction, according to the geometric relationship of the vector triangle $C O Q, \delta$ can be calculated as

$$
\delta=R_{1}-r_{1}\left(\theta_{1}\right)-\boldsymbol{e} \cdot \boldsymbol{r}_{0}
$$

in which, $r\left(\theta_{1}\right)$ is the radius vector of point $P$ in rotor coordinate system, and $\theta_{1}$ is the angle between $r$ and $z$ axis. $\boldsymbol{e}$ is the linear displacement vector from $O$ to $C$, named eccentricity, and it can be written as

$$
\boldsymbol{e}=e_{X} \boldsymbol{X}_{0}+e_{Y} \boldsymbol{Y}_{0}+e_{Z} \boldsymbol{Z}_{0}
$$

$r_{0}$ in eq. (3) is the unit vector of $r\left(\theta_{1}\right)$, and it can be described in stator coordinate system $\left(R_{1}, \theta, \varphi\right)$ as

$$
\boldsymbol{r}_{0}=\sin \theta \cos \varphi \boldsymbol{X}_{0}+\sin \theta \sin \varphi \boldsymbol{Y}_{0}+\cos \theta \boldsymbol{Z}_{0}
$$

where $\theta$ is the angle between $O P$ and $Z$-axis, and $\varphi$ is the azimuth of $O P$. Therefore, we can get

$$
\delta=R_{1}-r_{1}\left(\theta_{1}\right)-e_{X} \sin \theta \cos \varphi-e_{Y} \sin \theta \sin \varphi-e_{Z} \cos \theta
$$

In order to simplify the analysis, the rotor MPES with 
radius $r_{1}$ is assumed to be torispherical surface of revolution, and its radius vector in meridian plane can be described by Legendre polynomial series as

$$
r_{1}\left(\theta_{1}\right)=r_{10}+\sum_{n=1}^{\infty} a_{n} P_{n}\left(\cos \theta_{1}\right)
$$

where, $r_{10}$ is the nominal radius of the ideal envelope surface, $a_{n}$ is the aspheric coefficient of each harmonic in describing the envelope surface. $P_{n}\left(\cos \theta_{1}\right)$ is the Legendre polynomial series which is written as

$$
\begin{aligned}
& P_{1}\left(\cos \theta_{1}\right)=\cos \theta_{1} \\
& P_{2}\left(\cos \theta_{1}\right)=\frac{1}{2}\left(3 \cos ^{2} \theta_{1}-1\right) \\
& P_{n+1}\left(\cos \theta_{1}\right)=\frac{1}{n+1}\left[\begin{array}{l}
(2 n+1) \cos \theta_{1} P_{n}\left(\cos \theta_{1}\right) \\
-n P_{n-1}\left(\cos \theta_{1}\right)
\end{array}\right]
\end{aligned}
$$

Let $\delta_{0}=R_{10}-r_{10}, R_{10}$ is the nominal radius of the stator MPES with radius $R_{1}$. And substitute eq. (7) into (6), we can get

$$
\begin{aligned}
\delta= & \delta_{0}+\Delta \delta=\delta_{0}-\sum_{n=1}^{\infty}\left(a_{n}+b_{n}\right) P_{n}\left(\cos \theta_{1}\right) \\
& -e_{X} \sin \theta \cos \varphi-e_{Y} \sin \theta \sin \varphi-e_{Z} \cos \theta
\end{aligned}
$$

where

$$
\cos \theta_{1}=\alpha \sin \theta \cos \varphi+\beta \sin \theta \sin \varphi+\gamma \cos \theta
$$

And $b_{n}$ in eq. (9) is the aspheric coefficient of each harmonic in describing the stator envelope surface.

\subsection{Modeling of the drift error}

The magnetic force element on point $P$ can be expressed as $d F \cdot \boldsymbol{n}_{0}$, where $\boldsymbol{n}_{0}$ is the unit vector of normal line at point $P$. And the radius vector of $C P$ can be written as $r_{1} \cdot \boldsymbol{r}_{0}$. When the rotor MPES is an ideal sphere, $\boldsymbol{r}_{0}$ is coincided with $\boldsymbol{n}_{0}$, that is, the magnetic force passes through the geometric center of the rotor, and there is no disturbing torque about the geometric center. However, when the rotor MPES deviates from the ideal sphere and become a torispherical close surface, $\boldsymbol{r}_{0}$ and $\boldsymbol{n}_{0}$ are coincided no longer, disturbing torque is generated by the magnetic force about the geometric center of the rotor, which can be written in differential term as

$$
d \boldsymbol{T}=\boldsymbol{r} \times d \boldsymbol{F}=\left(r_{1} \cdot \boldsymbol{r}_{0}\right) \times\left(d F \cdot \boldsymbol{n}_{0}\right)
$$

So, the disturbing torque equation can be written as [14],

$$
\boldsymbol{T}=\iint_{S} \frac{\mu_{0} N^{2} I^{2}}{8 \delta_{0}^{2}}\left(1-\frac{2 \Delta \delta}{\delta_{0}}\right) \cdot\left(\frac{d r_{1}}{d \theta} \cdot \frac{1}{\sin \theta}\right) \cdot \boldsymbol{z}_{0} \times \boldsymbol{r}_{0} d S
$$

where $\mu_{0}$ is the magnetic permeability of vacuum, $N$ is the turns of coil on the stator magnetic pole, $I$ is the current in the coil, $S$ is the area of stator magnetic pole which applied magnetic force on the rotor.

Substituting eq. (1), (5), (7), (9) into (12), converting $d S$ to the spherical coordinate system, and calculating the disturbing torques generated from six stator magnetic bearings respectively, the final equation of the disturbing torques is as follow

$$
\begin{aligned}
\boldsymbol{T}= & \sum_{i=1}^{6} \iint_{S_{i}} \frac{\mu_{0} N^{2} I^{2} R_{i}^{2}}{8 \delta_{i 0}^{2}} \cdot\left(1-\frac{2 \Delta \delta_{i}}{\delta_{i 0}}\right) \cdot \sum_{n=1}^{\infty} a_{n} P_{n}^{\prime}\left(\cos \theta_{1}\right) \\
& \left.\cdot \begin{array}{c}
\beta \cos \theta-\gamma \sin \theta \sin \varphi \\
\gamma \sin \theta \cos \varphi-\alpha \cos \theta \\
\alpha \sin \theta \sin \varphi-\beta \sin \theta \cos \varphi
\end{array}\right] d \theta d \varphi
\end{aligned}
$$

in which $i$ denote the $i$ th magnetic pole of the stator, $R_{i}$ is the nominal radius of the $i$ th stator magnetic pole, $\delta_{i 0}$ is the nominal gap size between the $i$ th stator magnetic pole and the rotor, and $S_{i}$ is the area of the $i$ th stator magnetic pole.

Under the action of the disturbing torque, the spinning axis of rotor will gradually deviated from its original position, resulting drift angular velocity. According to angular momentum law, the drift angular velocity can be expressed as

$$
\left[\begin{array}{l}
\omega_{X} \\
\omega_{Y} \\
\omega_{Z}
\end{array}\right]=\frac{1}{H}\left[\begin{array}{ccc}
0 & \gamma & -\beta \\
-\gamma & 0 & \alpha \\
\beta & -\alpha & 0
\end{array}\right]^{-H}\left[\begin{array}{c}
T_{X} \\
T_{Y} \\
T_{Z}
\end{array}\right]
$$

where the angular momentum can be described with the polar moment of inertia of the rotor $J$ as,

$$
H=J \cdot \Omega
$$

\section{Torques from Assembly Errors of Stator}

\subsection{Torques from linear mismatch errors}

The linear mismatch errors refer to the misalignment in the axis direction of coordinate system $(X, Y, Z)$ when mounting the stator magnetic bearings. The errors changed the relative position of the rotor to the stator magnetic bearings, causing changes in the gap $\delta$ and the magnetic field distribution, and ultimately reflected in the generation of disturbing torque.

As is shown in Fig. 5, the linear displacement of stator magnetic bearing in $Y+$ direction can be written as

$$
\varepsilon=\varepsilon_{X} \boldsymbol{X}_{0}+\varepsilon_{Y} \boldsymbol{Y}_{0}+\varepsilon_{Z} \boldsymbol{Z}_{0}
$$

In the situation of linear mismatch errors, rules the gap $\delta$ changing is in correspond to subtracting the linear displacement $\varepsilon$ from the original eccentricity e. According 
to eq. (9), the gap size between the $Y+$ stator MPES and the rotor becomes

$$
\begin{aligned}
\delta^{\prime}= & \delta_{0}-\sum_{n=1}^{n} a_{n} P_{n}\left(\cos \theta_{1}\right)-\left(e_{X}-\varepsilon_{X}\right) \sin \theta \cos \varphi \\
& -\left(e_{Y}-\varepsilon_{Y}\right) \sin \theta \sin \varphi-\left(e_{Z}-\varepsilon_{Z}\right) \cos \theta
\end{aligned}
$$

Substituting eq. (17) into (12), the disturbing torque from the linear mismatch errors can be calculated as

$$
\begin{array}{r}
\frac{\mu_{0} N^{2} I^{2} R_{1}^{2}}{4 \delta_{0}^{3}} \cdot\left(\varepsilon_{x} \sin \theta \cos \varphi+\varepsilon_{y} \sin \theta \sin \varphi+\varepsilon_{z} \cos \theta\right) \\
\boldsymbol{T}_{l m_{-} Y_{+}+}=\iint_{S_{X_{+}}} \int_{n=1}^{\infty} a_{n} P_{n}^{\prime}\left(\cos \theta_{1}\right) \cdot\left[\begin{array}{c}
\beta \cos \theta-\gamma \sin \theta \sin \varphi \\
\gamma \sin \theta \cos \varphi-\alpha \cos \theta \\
\alpha \sin \theta \sin \varphi-\beta \sin \theta \cos \varphi
\end{array}\right] d \theta d \varphi
\end{array}
$$

Disturbing torque in other directions can be calculated in the same way, and the total disturbing torque generated from the linear mismatch errors can be obtained using eq. (13).

\subsection{Torques from asymmetry errors}

The pair of stator magnetic bearings which should be installed symmetrically does not achieve complete symmetrical installation, but slide a tiny angle from the ideal location in the envelope surface, which leads to the magnetic pole asymmetry errors. Dues to changes in magnetic pole location, the relative position of the rotor to the stator magnetic bearings is altered, so do the gap $\delta$ and the magnetic field distribution, resulting in disturbing torques.

In Fig. 5, the magnetic pole in the $Z+$ direction moved a deviation angle of $\zeta$ about the $X$-axis along the envelope surface, resulting in the asymmetry errors with the stator magnetic pole installed in $Z$ - direction.

In magnetic pole asymmetry errors, rule of the gap $\delta$ changing is similar to that of eccentricity $\mathbf{e}$ rotating $\zeta$ about

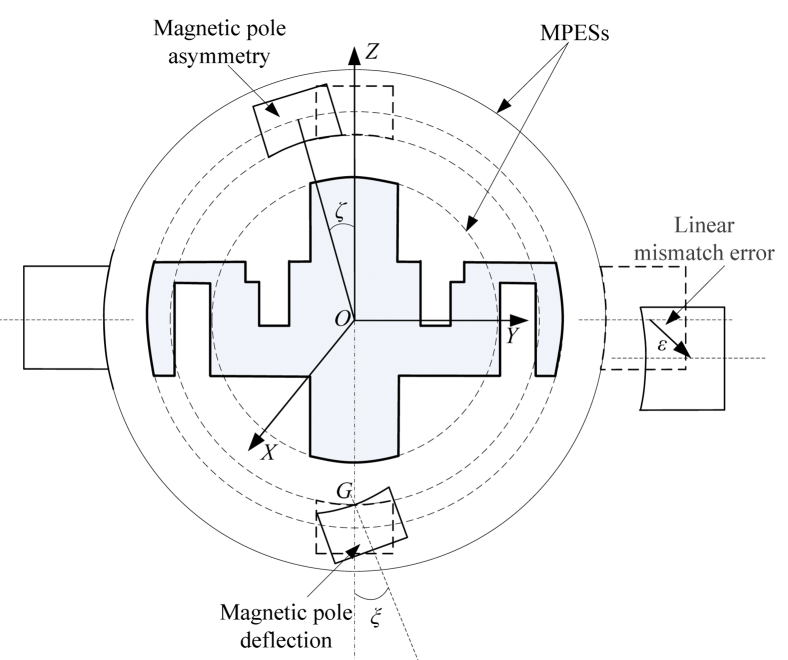

Fig. 5. (Color online) Stator errors of the MSSG. the $X$-axis. And the new eccentricity can be expressed as

$$
\boldsymbol{e}_{1}=\left[\begin{array}{c}
e_{X} \\
e_{Y} \cos \zeta+e_{Z} \sin \zeta \\
-e_{Y} \sin \zeta+e_{Z} \cos \zeta
\end{array}\right]
$$

Thus, gap size between the stator magnetic pole and the rotor can be written as

$$
\begin{aligned}
& \delta=-\sum_{n=1}^{n} a_{n} P_{n}\left(\cos \theta_{1}\right)-e_{X} \sin \theta \cos \varphi \\
& -\left(e_{Y} \cos \zeta+e_{Z} \sin \zeta\right) \sin \theta \sin \varphi-\left(e_{Z} \cos \zeta-e_{Y} \sin \zeta\right) \cos \theta
\end{aligned}
$$

Substituting eq. (20) into (12), we can get the disturbing torque generated from stator magnetic pole asymmetry errors in $Z+$ direction as

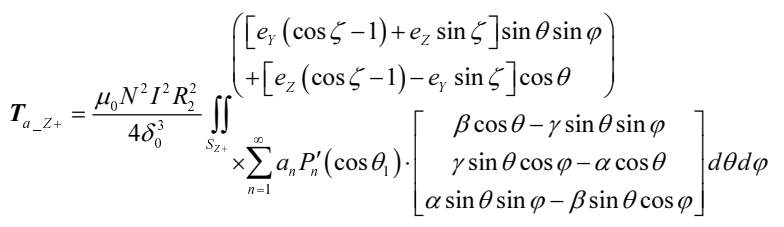

In eq. (21), the integral area needs to be divided into two parts when integrating,

$$
\begin{aligned}
& S_{Z+1}: \theta \in\left(0, \frac{\theta_{0}}{2}+\zeta\right), \quad \varphi \in\left(\begin{array}{ll}
0, & \pi
\end{array}\right) \\
& S_{Z+2}: \theta \in\left(0, \frac{\theta_{0}}{2}-\zeta\right), \quad \varphi \in\left(\begin{array}{ll}
0, & \pi
\end{array}\right)
\end{aligned}
$$

in which $\theta_{0}$ is the circle angle of stator in $O Y Z$-plane.

In general case, asymmetry errors can occur along the envelope surface in any direction. With $\Delta \theta_{X}, \Delta \theta_{Y}$ represent the deviation angles of an axial magnetic pole about the $X$ and Y-axis respectively, the eccentricity is converted to be

$$
\boldsymbol{e}_{2}=\left[\begin{array}{c}
e_{X} \cos \Delta \theta_{Y}-e_{Z} \sin \Delta \theta_{Y} \\
e_{X} \sin \Delta \theta_{X} \sin \Delta \theta_{Y}+e_{Y} \cos \Delta \theta_{X}+e_{Z} \sin \Delta \theta_{X} \cos \Delta \theta_{Y} \\
e_{X} \cos \Delta \theta_{X} \sin \Delta \theta_{Y}-e_{Y} \sin \Delta \theta_{X}+e_{Z} \cos \Delta \theta_{X} \cos \Delta \theta_{Y}
\end{array}\right]
$$

Substituting eq. (23) into (12), the disturbing torque generated from axial stator magnetic pole asymmetry errors can be written as

$$
\begin{aligned}
\boldsymbol{T}_{a_{-} Z_{+}}=\frac{\mu_{0} N^{2} I^{2} R_{2}^{2}}{4 \delta_{0}^{3}} \int_{S_{Z+}} & \left(\begin{array}{l}
{\left[e_{X}\left(\cos \Delta \theta_{Y}-1\right)-e_{Z} \sin \Delta \theta_{Y}\right] \sin \theta \cos \varphi} \\
+\left[\begin{array}{l}
e_{X} \sin \Delta \theta_{X} \sin \Delta \theta_{Y}+e_{Y}\left(\cos \Delta \theta_{X}-1\right) \\
+e_{Z} \sin \Delta \theta_{X} \cos \Delta \theta_{Y}
\end{array}\right] \sin \theta \sin \varphi \\
+\left[\begin{array}{l}
e_{X} \cos \Delta \theta_{X} \sin \Delta \theta_{Y}-e_{Y} \sin \Delta \theta_{X} \\
+e_{Z}\left(\cos \Delta \theta_{X} \cos \Delta \theta_{Y}-1\right)
\end{array}\right] \cos \theta
\end{array}\right) \\
\times \sum_{n=1}^{\infty} a_{n} P_{n}^{\prime}\left(\cos \theta_{1}\right) \cdot\left[\begin{array}{c}
\beta \cos \theta-\gamma \sin \theta \sin \varphi \\
\gamma \sin \theta \cos \varphi-\alpha \cos \theta \\
\alpha \sin \theta \sin \varphi-\beta \sin \theta \cos \varphi
\end{array}\right] d \theta d \varphi &
\end{aligned}
$$

Disturbing torque generated from the two pairs of stator magnetic bearings in radial can be calculated similarly using the above method. 


\subsection{Torques from deflection errors}

When the stator magnetic pole is deflected about its own vertex on envelope surface, the disturbing torque will be generated dues to the change of magnetic field distribution between the magnetic pole and the rotor.

In Fig. 5, the magnetic pole in the $Z$ - direction is deflected about its own vertex $G$ by $\xi$ counterclockwise in $X$-axis. It is equivalent to that the eccentricity e rotates about $G$ by $\xi$ counterclockwise in $X$-axis for the gap $\delta$. The eccentricity after rotation becomes

$$
\boldsymbol{e}_{3}=\left[\begin{array}{c}
e_{X} \\
e_{Y} \cos \xi+\left(e_{Z}+R_{1}\right) \sin \xi \\
-e_{Y} \sin \xi+\left(e_{Z}+R_{1}\right) \cos \xi-R_{1}
\end{array}\right]
$$

Substituting eq. (25) into (12), we can get the disturbing torque generated from magnetic pole deflection errors

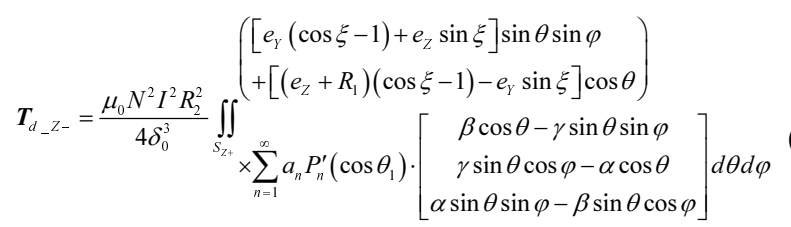

Generally, the stator magnetic pole can be deflected about its surface vertex in any direction. For the MSSG in this study, dues to the stator MPES in axial are symmetrical surfaces of revolution, deflections in Z-axis of which would not produce disturbing torques. But the deflections of other four radial magnetic poles in any direction will produce disturbing torques. Let $\Delta \lambda_{X}, \Delta \lambda_{Y}, \Delta \lambda_{Z}$ represent deflected angles of an magnetic pole about its own vertex in $X$-axis, $Y$-axis, and $Z$-axis respectively. For axial stator magnetic bearings, the eccentricity after deflection can be written as

$$
\boldsymbol{e}_{4}=\left[\begin{array}{c}
e_{X} \cos \Delta \lambda_{Y}-\left(e_{Z}-k R_{2}\right) \sin \Delta \lambda_{Y} \\
e_{Y} \cos \Delta \lambda_{X}+\left[e_{X} \sin \Delta \lambda_{Y}+\left(e_{Z}-k R_{2}\right) \cos \Delta \lambda_{Y}\right] \sin \Delta \lambda_{X} \\
-e_{Y} \sin \Delta \lambda_{X}+\left[e_{X} \sin \Delta \lambda_{Y}+\left(e_{Z}-k R_{2}\right) \cos \Delta \lambda_{Y}\right] \cos \Delta \lambda_{X}+k R_{2}
\end{array}\right]
$$

where $k$ is the coefficient of magnetic pole assembly, for the magnetic pole in axis positive direction, $k=1$, and for the negative direction $k=-1$. Substituting eq. (27) into (12), the disturbing torque generated from axial stator magnetic bearings can be obtained as

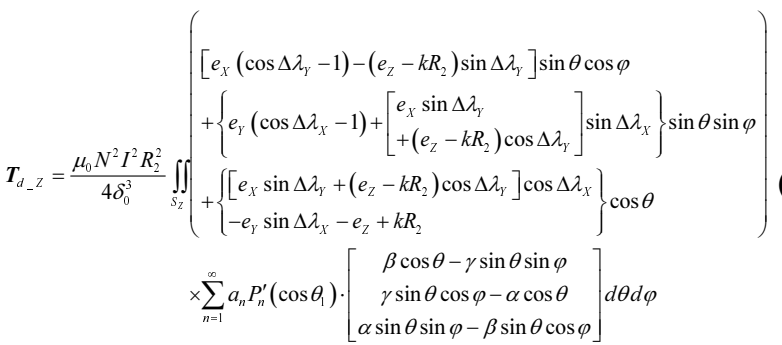

For the radial stator magnetic bearings in $X$ - and $Y$-axis, the disturbing torques from deflection can be calculated as

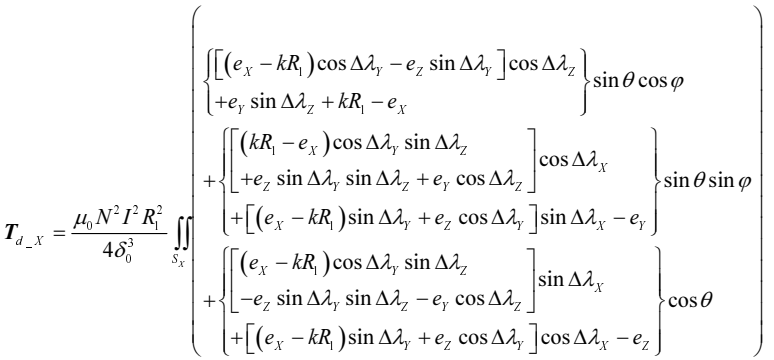

$$
\begin{aligned}
& \times \sum_{n=1}^{\infty} a_{n} P_{n}^{\prime}\left(\cos \theta_{1}\right) \cdot\left[\begin{array}{c}
\beta \cos \theta-\gamma \sin \theta \sin \varphi \\
\gamma \sin \theta \cos \varphi-\alpha \cos \theta \\
\alpha \sin \theta \sin \varphi-\beta \sin \theta \cos \varphi
\end{array}\right] d \theta d \varphi
\end{aligned}
$$

$$
\begin{aligned}
& \boldsymbol{T}_{d_{-} Y}=\frac{\mu_{0} N^{2} I^{2} R_{1}^{2}}{4 \delta_{0}^{3}} \iint_{S_{Y}}\left(\begin{array}{l}
\left\{\begin{array}{l}
\left.\left\{e_{X} \cos \Delta \lambda_{Y}-e_{Z} \sin \Delta \lambda_{Y}\right] \cos \Delta \lambda_{Z}\right\} \\
+\left(e_{Y}-k R_{2}\right) \sin \Delta \lambda_{Z}-e_{X}
\end{array}\right\} \sin \theta \cos \varphi \\
+\left\{\begin{array}{l}
e_{Z} \sin \Delta \lambda_{Y} \sin \Delta \lambda_{Z}-e_{X} \cos \Delta \lambda_{Y} \sin \Delta \lambda_{Z} \\
+\left(e_{Y}-k R_{2}\right) \cos \Delta \lambda_{Z} \\
+\left[e_{X} \sin \Delta \lambda_{Y}+e_{Z} \cos \Delta \lambda_{Y}\right] \sin \Delta \lambda_{X}+k R_{2}-e_{Y}
\end{array}\right\} \cos \Delta \lambda_{X}
\end{array}\right\} \sin \theta \sin \varphi \\
&+\left\{\begin{array}{l}
{\left[\begin{array}{l}
e_{X} \cos \Delta \lambda_{Y} \sin \Delta \lambda_{Z}-e_{Z} \sin \Delta \lambda_{Y} \sin \Delta \lambda_{Z} \\
-\left(e_{Y}-k R_{2}\right) \cos \Delta \lambda_{Z}
\end{array}\right] \sin \Delta \lambda_{X}} \\
+\left[e_{X} \sin \Delta \lambda_{Y}+e_{Z} \cos \Delta \lambda_{Y}\right] \cos \Delta \lambda_{X}-e_{Z}
\end{array}\right\} \cos \theta \\
& \times \sum_{n=1}^{\infty} a_{n} P_{n}^{\prime}\left(\cos \theta_{1}\right) \cdot\left[\begin{array}{c}
\beta \cos \theta-\gamma \sin \theta \sin \varphi \\
\gamma \sin \theta \cos \varphi-\alpha \cos \theta \\
\alpha \sin \theta \sin \varphi-\beta \sin \theta \cos \varphi
\end{array}\right] d \theta d \varphi
\end{aligned}
$$

\section{Case Study}

To simplify operations, the MSSG is assumed to working on a stabilized platform, so that $\alpha \approx 0, \beta \approx 0, \gamma \approx 1$. On the assumption that there is no magnetic flux leakage, errors of a single stator magnetic pole does not affect other poles. Take the $Y+$ stator magnetic pole of the MSSG studied in this paper for example, the integral area of the magnetic pole is $\theta \in\left(\frac{\pi}{2}-0.063, \frac{\pi}{2}+0.063\right), \varphi \in\left(\frac{\pi}{2}-0.5367, \frac{\pi}{2}+0.5367\right)$. Ignore the third-order trace and above, disturbing torques from different errors of stator are as follows,

$$
\begin{gathered}
\boldsymbol{T}_{l_{-} Y+}=\kappa\left[\begin{array}{ccc}
-0.0553 a_{1} & -0.0675 a_{1} & -0.0004 a_{2} \\
0.0675 a_{1} & 0.0553 a_{1} & 0.0004 a_{2} \\
0 & 0 & 0
\end{array}\right]\left[\begin{array}{c}
\varepsilon_{X} \\
\varepsilon_{Y} \\
\varepsilon_{Z}
\end{array}\right] \\
\boldsymbol{T}_{a_{-}{ }^{+}}=\kappa\left[\begin{array}{cc}
0.0553 a_{1} e_{Z}-0.0004 a_{2} e_{X} & -0.0553 a_{1} e_{Y}+0.0675 a_{1} e_{X} \\
-0.0675 a_{1} e_{Z}+0.0004 a_{2} e_{X} & -0.0553 a_{1} e_{X}+0.0675 a_{1} e_{Y} \\
0 & 0
\end{array}\right]\left[\begin{array}{c}
\Delta \theta_{X} \\
\Delta \theta_{Z}
\end{array}\right]
\end{gathered}
$$

where $\kappa=\frac{\mu_{0} N^{2} I^{2} R_{1}^{2}}{8 \delta_{0}^{3}}$.

In calculating the disturbing torque generated from deflection error, in consideration of the deflection angle is very small, we assumed that $\cos \Delta \lambda_{i} \approx 1,(i=X, Y, Z)$ 
and $\sin \Delta \lambda_{i} \approx \Delta \lambda_{i},(i=X, Y, Z)$, then the disturbing torque can be written as

$$
\boldsymbol{T}_{d_{-} Y+}=\kappa\left[\begin{array}{ccc}
-0.0675 a_{1} e_{Z} & 0.0553 a_{1} e_{Z} & 0.0044 a_{1}-0.0553 a_{1} e_{Y} \\
+0.0004 a_{2} e_{Y} & -0.0004 a_{2} e_{X} & +0.0675 a_{1} e_{X} \\
0.0553 a_{1} e_{Z} & -0.0675 a_{1} e_{Z} & -0.0053 a_{1}+0.0675 a_{1} e_{Y} \\
-0.0004 a_{2} e_{Y} & +0.0004 a_{2} e_{X} & -0.0553 a_{1} e_{X} \\
0 & 0 & 0
\end{array}\right]\left[\begin{array}{l}
\Delta \lambda_{X} \\
\Delta \lambda_{Y} \\
\Delta \lambda_{Z}
\end{array}\right]
$$

From the above equations of (37) to (39), we can see that the disturbing torques generated from stator magnetic bearings would apply to rotor in $X$ - and $Y$-axis, causing drifting of rotor spinning axis, but the disturbing torque in $Z$-axis is zero, thus has no affection on the spinning speed of rotor. The factor of rotor asphericity plays a decisive role in the generation of disturbing torque. When the envelope surfaces of rotor are ideal spherical, the disturbing force produced by the errors of stator will pass through the geometric center of rotor, and no disturbing torques appears. Eccentricity coupled with the aspheric coefficients of rotor is very important in eq. (38) and (39). Therefore, try to reduce the rotor asphericity and eccentricity, while take the rotor axes of inertia in the same direction of stator axes [13], the disturbing torque can be decreased significantly.

Figure 6 and Fig. 7 shows the relationship of the disturbing torque with rotor aspheric coefficients and the rotor eccentricity. From Fig. 6, it can be found that, the amplitude of the disturbing torque affected by rotor aspheric coefficients is about $10^{-10} \mathrm{~N} \cdot \mathrm{m}$. And as the increasing of $a_{1}$ and $a_{2}$, the value of disturbing torque increases significantly in $X$ - and $Y$ - direction, however, the increment of disturbing torque along with $a_{2}$ is far surpass that along with $a_{1}$. When the aspheric coefficients decrease to zero, the disturbing torque will disappear.

According to Fig. 7, we can discover that, the amplitude magnitude of the disturbing torque affected by the rotor eccentricity is the same to that of aspheric coefficients. The value of disturbing torque is increasing along with $e_{x}$ and $e_{y}$ in $X$ - and $Y+$ direction respectively. The increment of disturbing torque $T_{y}$ along with $e_{x}$ is obviously surpass that along with $e_{y}$. And the minimum value can be obtained if both $e_{x}$ and $e_{y}$ are zero.

Among all the errors of stator magnetic bearings, errors of linear mismatch is in correlation with the rotor asphericity, but independent of eccentricity in generating the disturbing torque, as in eq. (37). However, errors of linear mismatch, asymmetry, and deflection are all coupled with the aspheric coefficients of rotor, and the main source of the disturbing torque is the coupling with first harmonic, as is shown in eq. (37) to (39).

According to eq. (14), the drift velocity generated from stator magnetic pole errors in $Y+$ direction can be written as

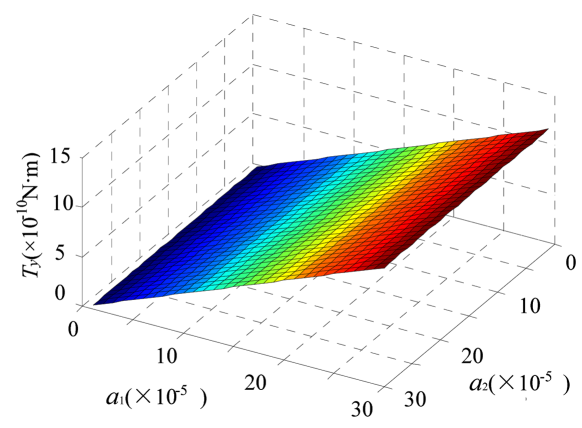

Fig. 6. (Color online) Relationship of disturbing torque and rotor aspheric coefficients.
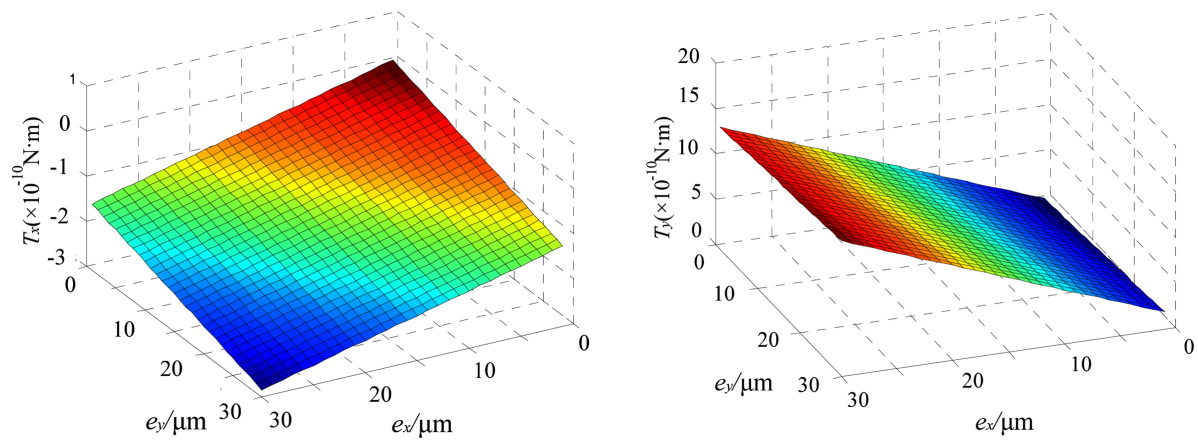

Fig. 7. (Color online) Relationship of disturbing torque and rotor eccentricity. 


$$
\left[\begin{array}{c}
\omega_{X} \\
\omega_{Y} \\
\omega_{Z}
\end{array}\right]=\frac{\kappa}{H}\left[\begin{array}{lll}
\mathbf{A} \boldsymbol{T}_{l_{-} Y+} & \mathbf{A} \boldsymbol{T}_{a_{-} Y+} & \mathbf{A} \boldsymbol{T}_{d_{-} Y+}
\end{array}\right]\left[\begin{array}{c}
c_{1} \\
c_{2} \\
\vdots \\
c_{8}
\end{array}\right]
$$

in which the error coefficient matrix $\kappa\left[\mathbf{A} \boldsymbol{T}_{l_{Y+}} \mathbf{A} \boldsymbol{T}_{a_{Y+}} \mathbf{A} \boldsymbol{T}_{d_{-}+}\right]$ is the combination of each error coefficient matrix in eq. (37) to (39), in the error matrix $\left[\begin{array}{llll}c_{1} & c_{2} & \ldots & c_{8}\end{array}\right]^{\mathrm{T}}, c_{i}$ is defined as,

$$
\left[\varepsilon_{X} \varepsilon_{Y} \varepsilon_{Z} \Delta \theta_{X} \Delta \theta_{Z} \Delta \lambda_{X} \Delta \lambda_{Y} \Delta \lambda_{Z}\right]^{\mathrm{T}}
$$

Assume the linear mismatch is $10 \mu \mathrm{m}$, and the angular errors are $0.001^{\circ}, a_{1}=12.392 \times 10^{-6}, a_{2}=14.424 \times 10^{-6}$, the final drift angular velocity can be calculated as

$$
\left[\begin{array}{c}
\omega_{X} \\
\omega_{Y} \\
\omega_{Z}
\end{array}\right]=\left[\begin{array}{c}
-0.1642 \times 10^{-60} / \mathrm{h} \\
0.1613 \times 10^{-60} / \mathrm{h} \\
0
\end{array}\right]
$$

Owning to the limitation of space, we only analyzed the drift angular velocity generation mechanism of stator magnetic pole in $Y+$ direction, just as shown in eq. (42), however, the drift angular velocity generated from other stator magnetic bearings can be calculated in the same way, and the general drift angular velocity produced by the errors of stator is the sum of drift angular velocities generated from all six stator magnetic bearings.

\section{Conclusion}

According to the physical mechanism of a dual spherical envelope surface MSSG, the disturbing torque generated from stator assembly errors is analyzed. Analytical models of the disturbing torque have been founded. According to the analytical models, the disturbing torque and the drift angular velocity of the stator magnetic pole in $Y+$ direction have been discussed particularly. Theoretical analysis shows that the error of rotor asphericity is the most critical factor leading to disturbing torques. And it is the fundamental cause of disturbing torque that the error of rotor asphericity is coupled with various errors of the stator. Therefore, it is an important means to reduce the drift angular velocity that guaranteeing the rotor envelope surface to be an ideal spherical when it spinning steadily by increasing the machining precision. The rotor eccentricity coupled with the errors of asymmetry and deflection is another important cause of disturbing torque. The first harmonic of rotor asphericity corresponds to the translation of rigid body axially, which is the main source of disturbing torques from linear mismatch by coupled with it. Theoretically, drift velocity introduced by factors mentioned above can be significantly decreased by controlling the rotor spinning at the center of the stator cavity.

The research work of this paper achieved the drift angular velocity generation mechanism of stator magnetic bearing in a kind of MSSG, and expanded the understanding of the disturbing torques generated from the errors of stator. What we discussed in this paper will provide an available reference for the structure optimization, drift angular velocity measurement and error compensation of a MSSG.

\section{References}

[1] J. Q. Tang, C. E. Wang, and X. Biao, J. Magn. 18, 432 (2013).

[2] C. H. Park, S. K. Choi, J. H. Ahn, S. Y. Ham, and S. Kim, J. Magn. 18, 302 (2013).

[3] Y. Ren and J. C. Fang, IEEE Trans. Ind. Electron. 61, 1539 (2014).

[4] Y. Ren and J. C. Fang, Math. Probl. Eng. 2014, 1 (2014).

[5] Y. Ren, J. C. Fang, and Y. H. Fan, IEEE Trans. Ind. Electron. 61, 2003 (2014).

[6] Y. Maruyama, T. Mizuno, M. Takasaki, Y. Ishino, T. Ishigami, and H. Kameno, J. Syst. Des. and Dyn. 2, 155 (2008).

[7] Y. Maruyama, T. Mizuno, M. Takasaki, Y. Ishino, and H. Kameno, J. Mechatronics. 19, 1261 (2009).

[8] Y. Maruyama, T. Mizuno, M. Takasaki, Y. Ishino, H. Kameno, and A. Kubo, Industrial Electronics, 35th Annual Conference of IEEE 61, 1911 (2009).

[9] J. C.Fang, S. Q. Zheng, and B. C. Han, IEEE Trans. Instrum. Meas. 61, 338 (2012).

[10] X. N. Hu, Q. L. Wang, C. Y. Cui, F. Gao, H. Wang, Y. Li, H. S. Wang, J. S. Cheng, Y. M. Dai, and L. G. Yan, IEEE Trans. Instrum. Meas. 63, 2789 (2014).

[11] C. He and Q. L. Wang, J. Cry. 47, 413 (2007).

[12] C. He, Q. L. Wang, and X. Li, IEEE Trans. Appl. Supercond. 20, 47 (2010).

[13] C. He, Q. L. Wang, C. Li, L. Yan, and Y. Dai, IEEE Trans. Appl. Supercond. 17, 2174 (2007).

[14] C. Y. Cui, X. N. Hu, J. S. Cheng, H. Wang, and Q. L. Wang, Acta Phys. Sin. 64, 018403 (2015). 